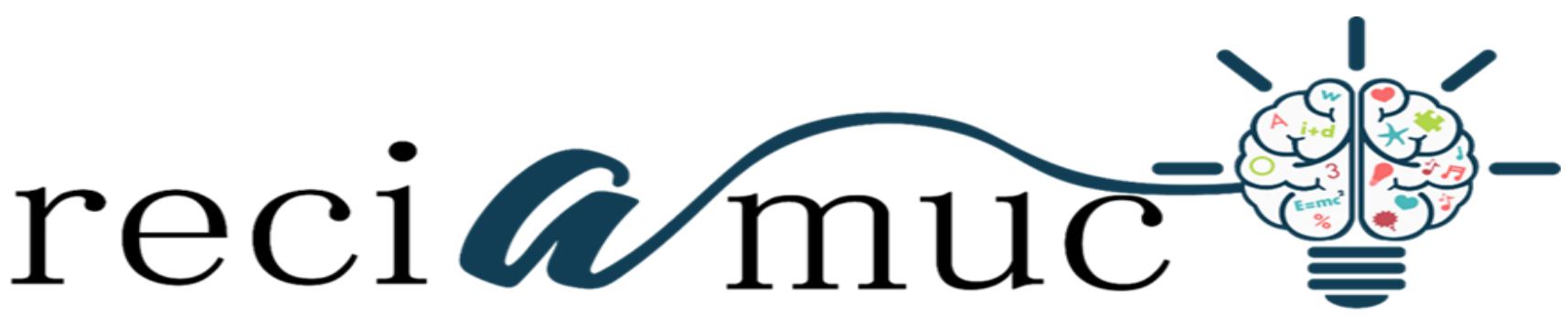

Revista cientifica de investigación actualización del mundo de las ciencias

José Ignacio Macías Alcívar a ; Jenny Marisela Briones Fajardo ${ }^{\text {b; }}$ Giannella Lissette Ramírez López ${ }^{\text {c; }}$ Edwing Xavier Pincay Macías d

Enfermedad pulmonar obstructiva crónica (EPOC)

Chronic obstructive pulmonary disease (COPD)

Revista Científica de Investigación actualización del mundo de las Ciencias. Vol. 3 núm., 1, enero, ISSN: 2588-0748, 2018, pp. 95-126

DOI: $10.26820 /$ reciamuc/3.(1).enero.2019.95-126

URL: $\underline{\text { htp: }: / / \text { reciamuc.com/index.php/RECIAMUC/article/view/225 }}$

Código UNESCO: 3205 Medicina Interna

Tipo de Investigación: Artículo de Revisión

Editorial Saberes del Conocimiento

Recibido: 10/12/2018

Aceptado: 20/01/2019

Publicado: 30/01/2019

Correspondencia: director@reciamuc.com
a. Médico; joseig.89escritor@hotmail.com; Guayaquil, Ecuador.
b. Médico; jeimmy_b@hotmail.com; Guayaquil, Ecuador
c. Médico; génesis-alava@ outllook.es; Guayaquil, Ecuador
d. Médico; edwingxpm@gmail.com; Guayaquil, Ecuador 


\section{Enfermedad pulmonar obstructiva crónica (EPOC)}

Vol. 3, núm. 1., (2019)

José Ignacio Macías Alcívar; Jenny Marisela Briones Fajardo; Giannella Lissette Ramírez López; Edwing Xavier Pincay Macías

\section{RESUMEN}

El sistema respiratorio es fundamental para el equilibrio bioquímico y físico del organismo. Este se encarga de inhalar el oxígeno y otros gases que están presentes en el medio ambiente y, en condiciones normales, son tratados y expulsados hacia el exterior del cuerpo. Cuando esta funcionabilidad no ocurre entonces hay presencia de insuficiencia respiratoria lo cual determina una enfermedad en el sistema respiratorio. Una de estas enfermedades en la enfermedad pulmonar obstructiva crónica (EPOC) que no es más que la limitación del flujo aéreo que no permite la inhalación-exhalación normal por lo que se manifiesta en tos, disnea y expectación. El objetivo general de esta investigación es analizar las características del EPOC. La metodología empleada se basó en una investigación de tipo documental y bibliográfica. Los resultados se basaron en los síntomas, causas, diagnóstico y tratamiento de la EPOC. Como conclusión se determinó que las causas principales son la inhalación de humo de leña y de tabaco. Los diagnósticos establecidos están determinados principalmente por inspección física del paciente para luego aplicar espirómetro como método principal de detección, seguidamente de métodos secundarios como radiografías TAC, electrocardiograma, ecocardiograma, entre otros. El tratamiento va depender de varios factores como tratamientos no farmacológicos como planes educativos, método de dejar el cigarrillo, autocontrol, ayudas psicológicas, actividad física y vacunas. También están los tratamientos farmacológicos que empiezan con medicamentos que ayudan a sustituir al tabaco para luego realizar tratamiento según sea la gravedad.

Palabras Claves: Sistema Respiratorio; Enfermedad Respiratoria; EPOC; Espirometría. 


\title{
Enfermedad pulmonar obstructiva crónica (EPOC)
}

Vol. 3, núm. 1., (2019)

José Ignacio Macías Alcívar; Jenny Marisela Briones Fajardo; Giannella Lissette Ramírez

López; Edwing Xavier Pincay Macías

\begin{abstract}
The respiratory system is fundamental for the biochemical and physical balance of the organism. This is responsible for inhaling oxygen and other gases that are present in the environment and, under normal conditions, are treated and expelled to the outside of the body. When this functionality does not occur then there is presence of respiratory insufficiency which determines a disease in the respiratory system. One of these diseases in chronic obstructive pulmonary disease (COPD) is nothing more than the limitation of airflow that does not allow normal inhalation-exhalation so it manifests in cough, dyspnea and expectation. The general objective of this research is to analyze the characteristics of COPD. The methodology used was based on documentary and bibliographic research. The results were based on the symptoms, causes, diagnosis and treatment of COPD. In conclusion, it was determined that the main causes are the inhalation of wood smoke and tobacco smoke. The established diagnoses are determined mainly by physical inspection of the patient to then apply spirometry as the main method of detection, followed by secondary methods such as CAT scans, electrocardiogram, echocardiogram, among others. The treatment will depend on several factors such as non-pharmacological treatments such as educational plans, method of quitting, self-control, psychological aids, physical activity and vaccines. There are also pharmacological treatments that start with medications that help replace the tobacco and then perform treatment depending on the severity.
\end{abstract}

Key Words: Respiratory System; Respiratory Disease; COPD; Spirometry. 


\section{Enfermedad pulmonar obstructiva crónica (EPOC)}

Vol. 3, núm. 1., (2019)

José Ignacio Macías Alcívar; Jenny Marisela Briones Fajardo; Giannella Lissette Ramírez López; Edwing Xavier Pincay Macías

\section{Introducción.}

Para obtener una excelente calidad de vida de debe tener una buena alimentación y ejercitación a través de varios mecanismos de preparación física. Para poder realizar las actividades físicas es necesario tener una buena respiración. Para ello el organismo se poya en el sistema respiratorio que es capaz de transformar un elemento gaseoso en otro con la finalidad de producir la energía necesario que requiere el cuerpo para movilizarse, hacer las funciones cotidianas entre otro.

Es por ello que es necesario poder comprender la importancia del sistema respiratorio para el organismo. Es el intercambio de gases entre la atmósfera y la sangre, donde el oxígeno (O2) es introducido dentro del cuerpo para su posterior distribución a los tejidos y el dióxido de carbono (CO2) producido por el metabolismo celular, es eliminado al exterior(Reiriz Palacios, 2019). Esto se puede observar en la Figura 1.

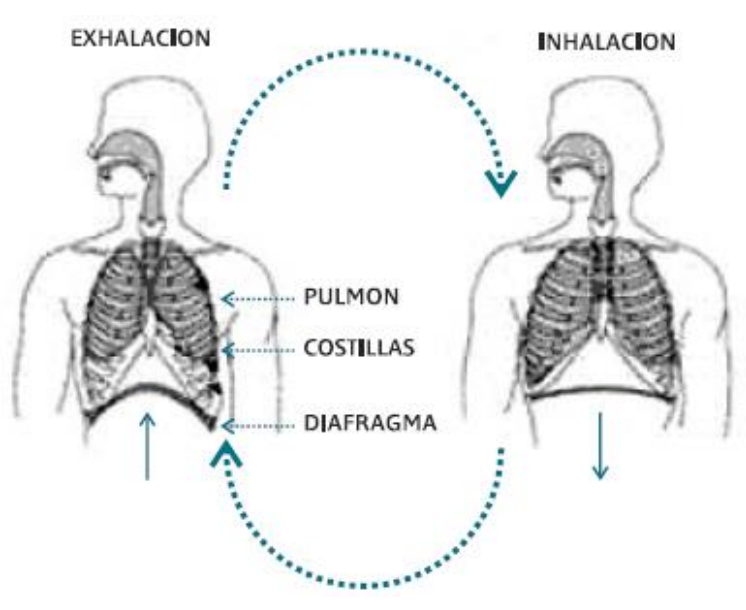

Figura 1. Sistema respiratorio

Fuente: (UNNE, 2019) 


\section{Enfermedad pulmonar obstructiva crónica (EPOC)}

Vol. 3, núm. 1., (2019)

José Ignacio Macías Alcívar; Jenny Marisela Briones Fajardo; Giannella Lissette Ramírez López; Edwing Xavier Pincay Macías

Además de esta funcionabilidad estas planteado otras características de este sistema lo que permite que el sistema sea más complejo de lo habitual. El respirar nos da otras funciones como:

Ventilación alveolar, que es el intercambio de aire entre la atmósfera y los alvéolos pulmonares, y viceversa; hematosis o intercambio de gases entre los alvéolos pulmonares y la sangre del capilar pulmonar; transporte de gases, que se realiza a través de la sangre; y difusión de gases entre la sangre y las células a nivel tisular. (UNNE, 2019, pág. 41)

El sistema puede inhalar una serie de gases, partículas y vapores que el organismo, en condiciones de equilibrio estable, puede combatir, transformar y expulsar hacia el medio ambiente.

En condiciones normales, los gases inhalados son destoxificados, las toxinas son neutralizadas; las partículas son atrapadas y eliminadas, y los microorganismos son atrapados, destruidos y eliminados. Cada región anatómica-histológica del aparato respiratorio tiene su propio mecanismo de defensa. En otras palabras, el sistema de conducción (de nariz a bronquios), sistema de transición (bronquiolos) y sistema de intercambio (alvéolos) tiene cada uno un mecanismo diferente de defensa.(López, 2006, pág. 3)

Es necesario entonces poder desarrollar la evolución del sistema respiratorio desde la etapa embrionaria hasta la etapa alveolar debido a la formación de organismos que cumplen las diferentes funciones del sistema. La etapa embrionaria ocurre en las 5 primeras semanas postconcepción donde el pulmón se desarrolla desde el intestino primitivo anterior en la cuarta semana de gestación y el surco laringotraqueal se desarrolla del endodermo anterior a los 26 días 


\section{Enfermedad pulmonar obstructiva crónica (EPOC)}

Vol. 3, núm. 1., (2019)

José Ignacio Macías Alcívar; Jenny Marisela Briones Fajardo; Giannella Lissette Ramírez López; Edwing Xavier Pincay Macías

de gestación(Sánchez, 2001). Durante este periodo se originan las vías aéreas de mayor calibre, a partir de la formación braqueal y su primera dicotomisación que formara la carina (T4) y eventualmente los bronquios fuentes derecho e izquierdo hasta los 18 segmentos lobares (Asenjo, 2017). Esto se puede detallar en la Figura 2 donde se puede visualizar: (A) y (B) la formación de la vasculatura pulmonar, cartílago, musculo liso y tejido conectivo paralelamente; y (C) la formación de los bronquios fuentes derecho e izquierdo.

(A)

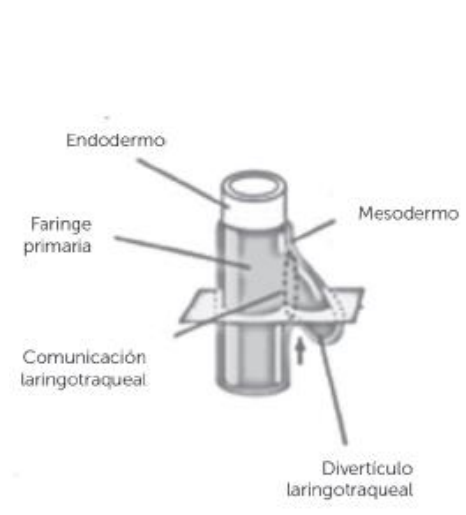

(B)

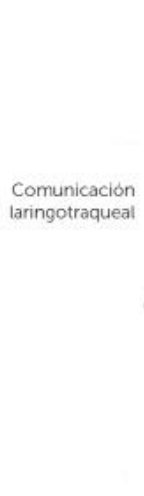

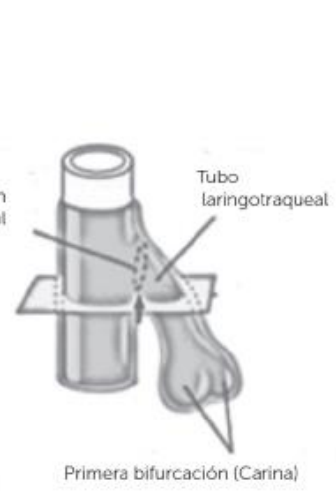

(C)

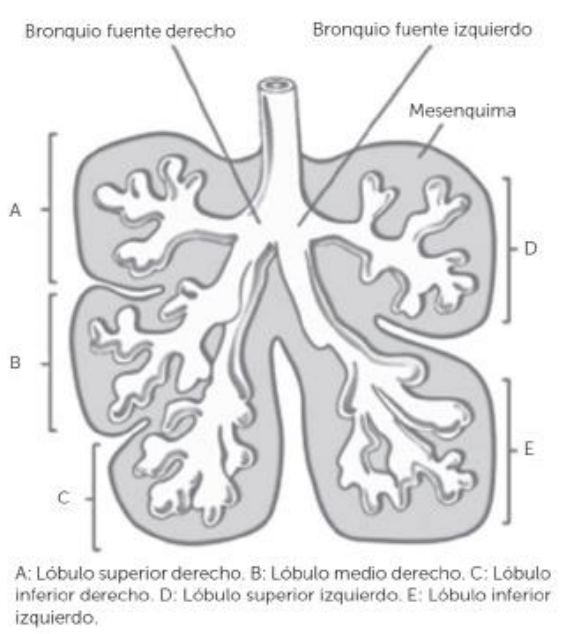

Figura 2. Etapa embrionaria. (A) y (B) la formación de la vasculatura pulmonar, cartílago, musculo liso y tejido conectivo paralelamente; y (C) la formación de los bronquios fuentes derecho e izquierdo

Fuente: (Asenjo, 2017)

La etapa pseudoglandular se forma a partir de la 5ta y 16va semana de la gestación donde se desarrollan las vías aéreas. Es vital en este período la relación entre endodermo y mesodermo, ya que todas las ramas de la porción conductora del árbol traqueobronquial, desde la tráquea 


\section{Enfermedad pulmonar obstructiva crónica (EPOC)}

Vol. 3, núm. 1., (2019)

José Ignacio Macías Alcívar; Jenny Marisela Briones Fajardo; Giannella Lissette Ramírez López; Edwing Xavier Pincay Macías

hasta los bronquiolos terminales, quedan establecidos, posterior a este período, no se forman nuevas ramas (Sánchez, 2001). Esto se puede ver en la Figura 3(A).

La etapa canalicular se forma entre las 16 a 24 semanas de gestación y se caracteriza por la proliferación de la irrigación sanguínea de la mesénquima. Los capilares se acercan a la vía aérea, con un progresivo aplanamiento del epitelio respiratorio con lo cual, al término de este período, se hace posible la respiración (Sánchez, 2001). Es decir, se produce u adelgazamiento epitelial para entrar en íntimo contacto con el lecho capilar cercano, a partir de las células de revestimiento alveolar tipo II (neumocitos tipo II) para originar los neumocitos Tipo I en este proceso (Asenjo, 2017). Esto se puede visualizar en la Figura 3(B).

La etapa sacular se desarrolla en el período entre la 28va y 36va semana. A partir de los bronquios terminales se generan 3 dicotomizaciones que dan origen a los bronquiolos respiratorios y estos a su vez 3 sáculos terminales que aumentan la superficie de intercambio gaseoso adelgazando su pared hasta formar los septos primarios (Asenjo, 2017). En estos septos primarios se deposita una fibra elástica donde se formarán más adelante los septos secundarios. Esto se puede observar en la Figura 3(C).

La etapa alveolar se forma en el período final de gestación donde sucede la división de los sáculos. Formación de los septos secundarios a partir de una doble esa capilar separada por una vaina de tejido conectivo (maduración microvascular), como también hiperplasia alveolar hasta los 3 años y posterior hipertrofia o aumento de tamaño alveolar hasta los 8 años (Asenjo, 2017). Esto se muestra en la Figura 3 (D). 


\section{Enfermedad pulmonar obstructiva crónica (EPOC)}

Vol. 3, núm. 1., (2019)

José Ignacio Macías Alcívar; Jenny Marisela Briones Fajardo; Giannella Lissette Ramírez López; Edwing Xavier Pincay Macías

Ahora, existen diferencias entre la estructura anatómica del aparato respiratorio de los recién nacidos, niños y adultos los cuales están divididos en vía aérea alta y baja. Existe una relación a la resistencia de la vía aérea, existe un aumento del diámetro relativo de la vía aérea con el crecimiento, lo que hace que disminuya la resistencia en los primeros años de la vida (Sánchez, 2001). En la Tabla 1 se detalla las características anatómicas del aparato respiratorio del lactante. Las Figura 4 y Figura 5, se pueden ver las diferentes estructuras que corresponden al aparato respiratorio, vía área alta y baja respectivamente.

(A)

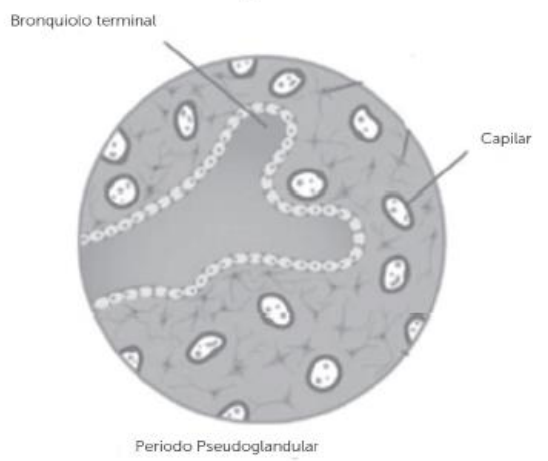

(C)

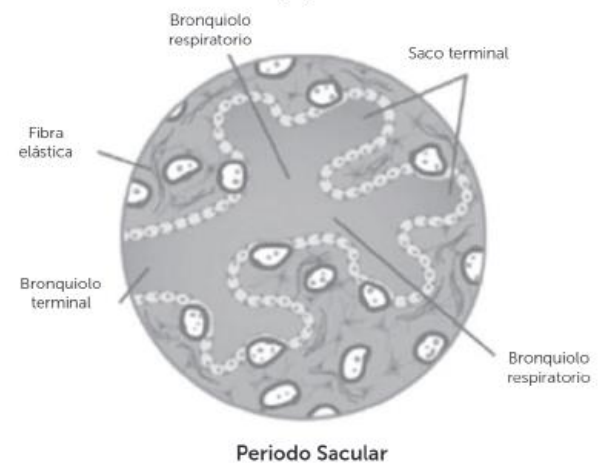

(B)

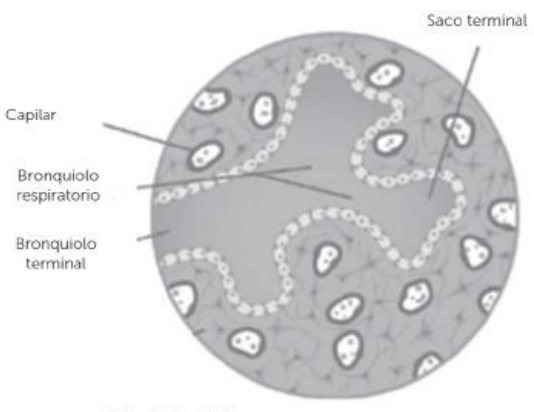

(D)

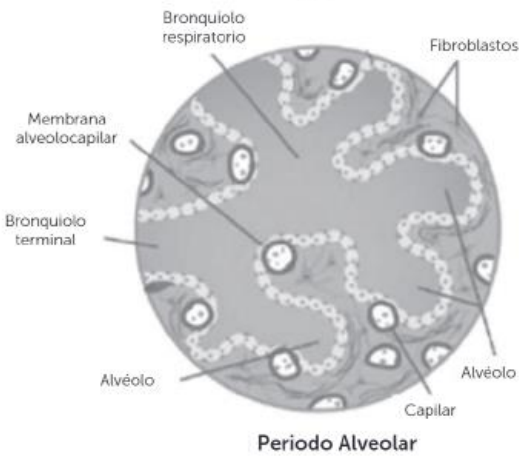

Figura 3. Formación del sistema respiratorio. (A) Etapa pseudoglandular, (B) etapa canalicular, (C) etapa sacular y (D) etapa alveolar

Fuente: (Asenjo, 2017) 


\section{Enfermedad pulmonar obstructiva crónica (EPOC)}

Vol. 3, núm. 1., (2019)

José Ignacio Macías Alcívar; Jenny Marisela Briones Fajardo; Giannella Lissette Ramírez

López; Edwing Xavier Pincay Macías

\section{Tabla 1. Características anatómicas del aparato respiratorio del lactante}

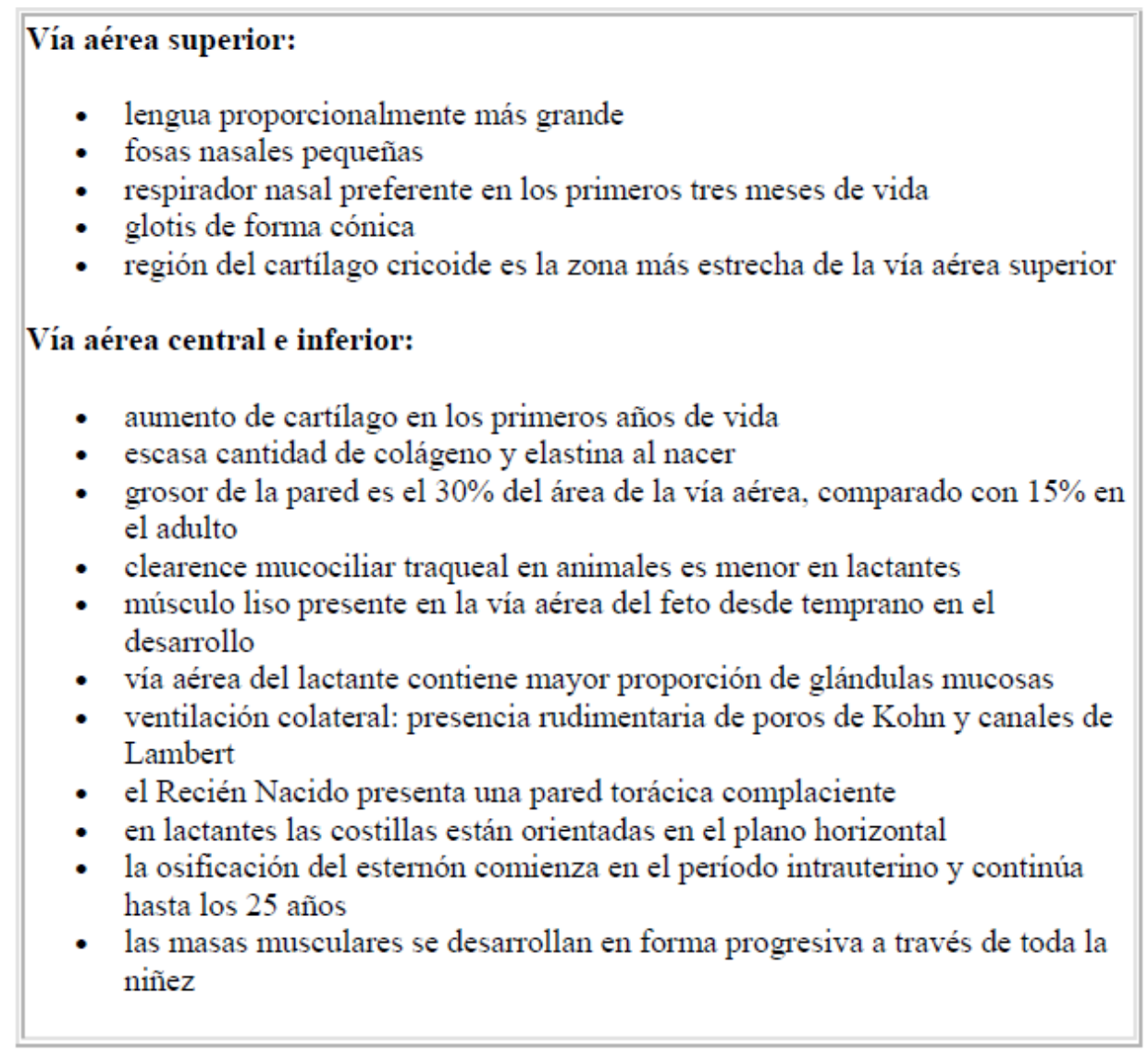

Fuente: (Sánchez, 2001) 


\section{Enfermedad pulmonar obstructiva crónica (EPOC)}

Vol. 3, núm. 1., (2019)

José Ignacio Macías Alcívar; Jenny Marisela Briones Fajardo; Giannella Lissette Ramírez López; Edwing Xavier Pincay Macías

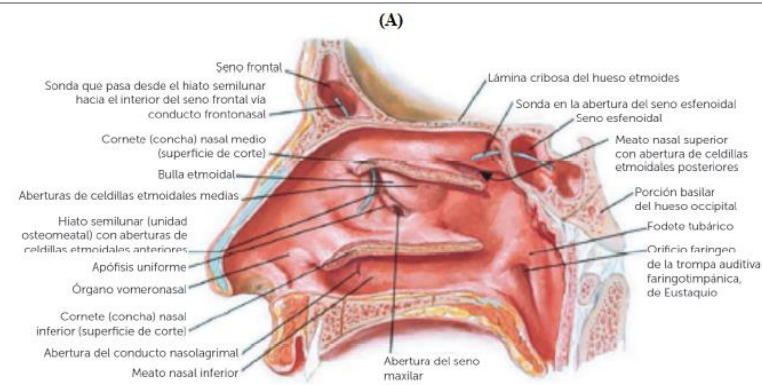

(B)

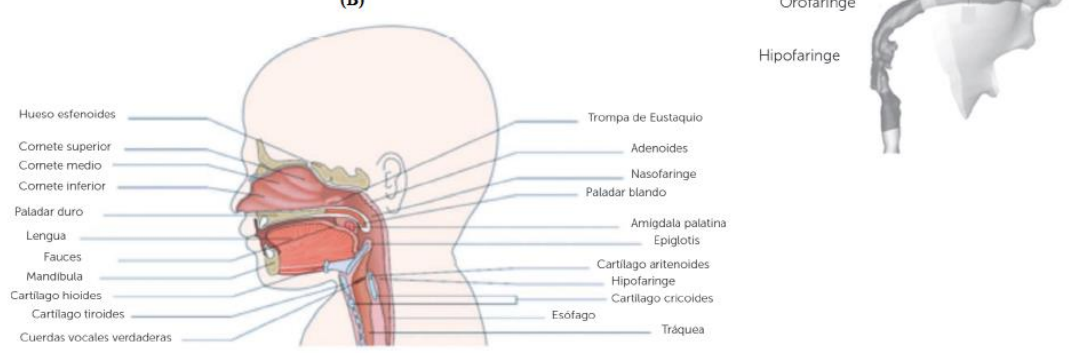

Figura 4. Aparato respiratorio de la vía aérea alta. (A) Nariz y fosas nasales, (B) cavidad oral y lengua y (C) faringe

Fuente: (Asenjo, 2017) 


\section{Enfermedad pulmonar obstructiva crónica (EPOC)}

Vol. 3, núm. 1., (2019)

José Ignacio Macías Alcívar; Jenny Marisela Briones Fajardo; Giannella Lissette Ramírez

López; Edwing Xavier Pincay Macías

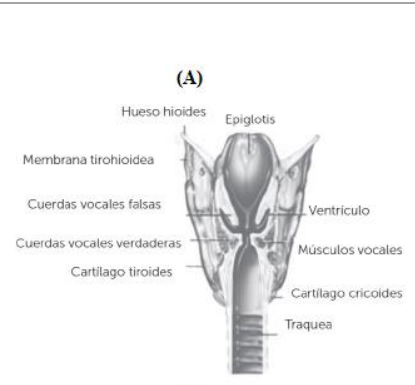

(C)
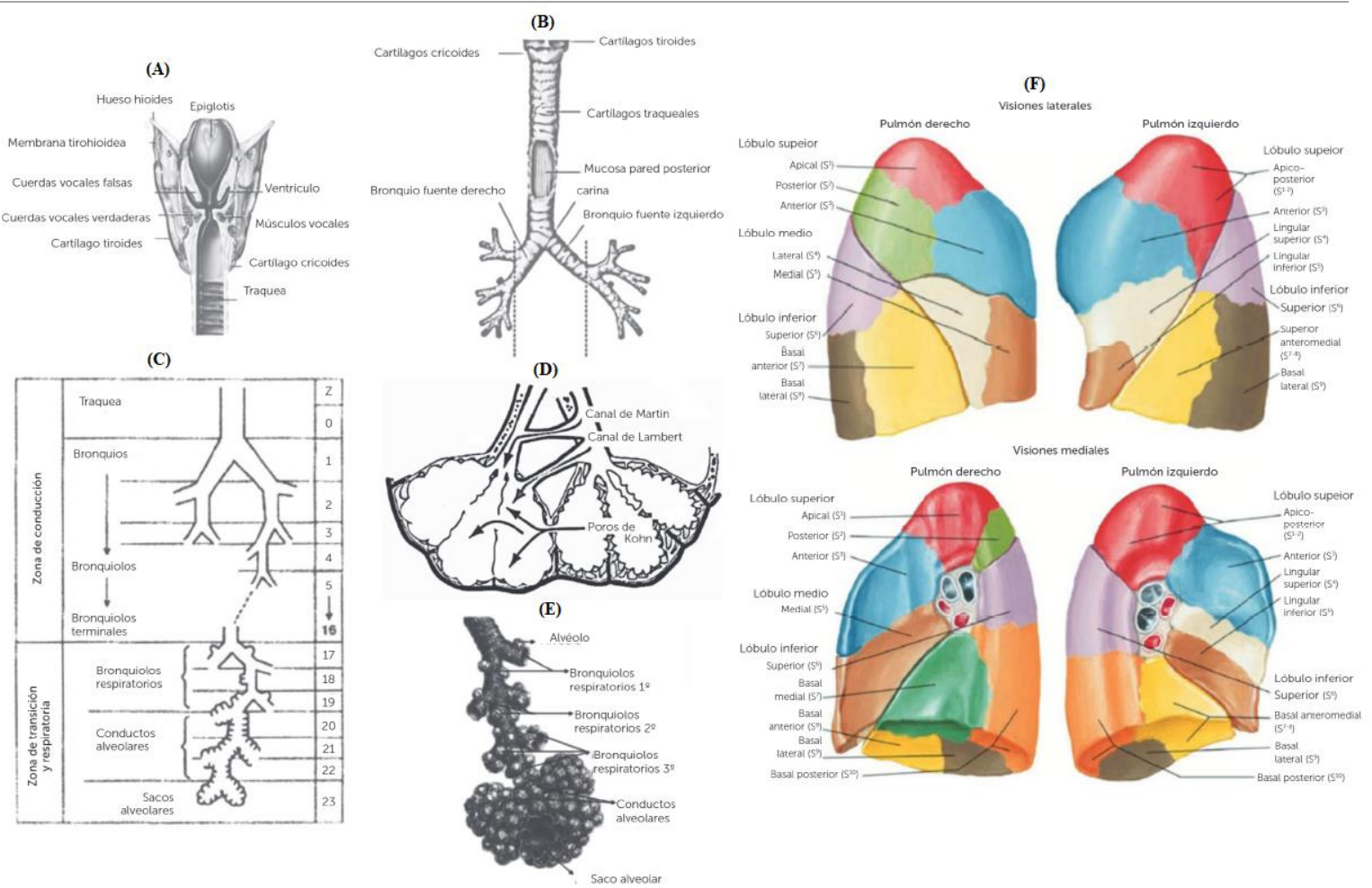

Figura 5. Aparato respiratorio de la vía aérea baja. (A) Laringe, (B) tráquea, (C) bronquios, (D) bronquiolos, (E) alveolos y (F) pulmón

Fuente: (Asenjo, 2017)

Al tener un buen aparato respiratorio se podrá entonces mantener la integridad estructural y funcional del sistema a través de inhalación de oxígeno al organismo. La enfermedad respiratoria se caracteriza precisamente por un compromiso en la utilización eficaz del oxígeno, por lo cual dependerá de la variedad clínica que adopte esta insuficiencia (Díaz, y otros, 2003).

Es por ello la importancia del análisis de las enfermedades del sistema respiratorio porque con ella se puede afectar el funcionamiento de algunas miembros del aparato que participan en la 


\section{Enfermedad pulmonar obstructiva crónica (EPOC)}

Vol. 3, núm. 1., (2019)

José Ignacio Macías Alcívar; Jenny Marisela Briones Fajardo; Giannella Lissette Ramírez López; Edwing Xavier Pincay Macías

respiración. En la Tabla 2 se muestran la clasificación de las enfermedades del sistema respiratorio.

Tabla 2. Clasificación de las enfermedades del sistema respiratorio

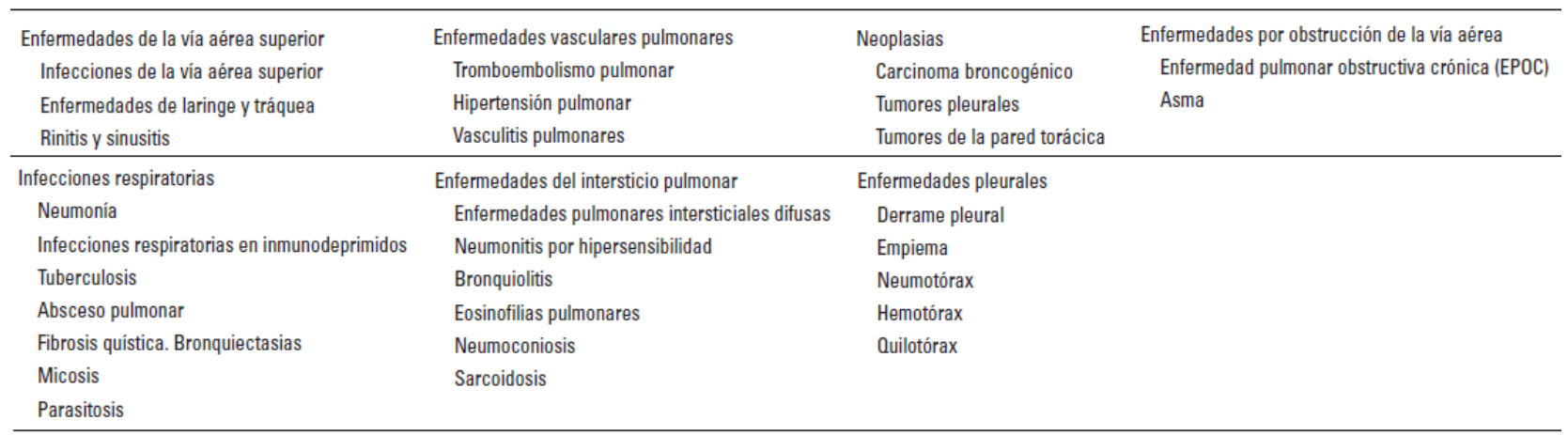

Fuente: (Galera, Carrera, \& Ortega, 2010)

Muchas de estas enfermedades son causadas por una insuficiencia respiratoria. Esta carencia la sufren, en su mayoría, la población pediátrica. Las causas de la insuficiencia respiratoria se pueden observar en la Tabla 3. La insuficiencia respiratoria resulta cuando la función de bomba de los músculos respiratorios es insuficiente para mantener un nivel de intercambio gaseoso a nivel alveolar que supla las necesidades metabólicas de la respiración celular (Quiroga, Zúñiga, Martínez, \& Mantilla, 2008).

También existen variables que pueden afectar a la respiración. Estos parámetros se encuentran dentro de las condiciones y forma de vida del individuo. Una de ello es la condición ambiental y laboral de la persona. El asma ocupacional representó el grupo diagnóstico más frecuente, seguido de las enfermedades pleurales no malignas y dentro de éstas las placas 


\section{Enfermedad pulmonar obstructiva crónica (EPOC)}

Vol. 3, núm. 1., (2019)

José Ignacio Macías Alcívar; Jenny Marisela Briones Fajardo; Giannella Lissette Ramírez López; Edwing Xavier Pincay Macías

pleurales calcificadas, en el tercer lugar lo ocupaban las neumoconiosis y en el cuarto la alveolitis alérgica extrínseca y el mesotelioma(Abú Shams, y otros, 2005).

\section{Tabla 3. Causas de insuficiencia respiratoria}

\begin{tabular}{|c|c|}
\hline Mecanismo & Enfermedades desencadenantes de insuficiencia respiratoria \\
\hline Inadecuada captación de $\mathrm{O}_{2}$ & $\begin{array}{l}\text { - Neoplasias } \\
\text { - Infecciones virales, bacterianas, micóticas } \\
\text { - Traumatismos: contusión pulmonar } \\
\text { - Enfermedades obstructivas: EPOC, asma } \\
\text { - Insuficiencia cardiaca } \\
\text { - Embolia pulmonar } \\
\text { - Atelectasias }\end{array}$ \\
\hline Inadecuado intercambio de $\mathrm{CO}_{2}$ & $\begin{array}{l}\text { - Medicamentos: opioides, benzodiacepinas, anestésicos } \\
\text { - Alteraciones metabólicas: hiperglicemia, hipofosfatemia, hiponatremia, hipocalce- } \\
\text { mia } \\
\text { - Infecciones del SNC: meningitis, encefalitis } \\
\text { - Malformaciones de la caja torácica: cifoescoliosis, espondilitis } \\
\text { - Otras: hipoventilación alveolar central, apnea obstructiva del sueño }\end{array}$ \\
\hline Alteraciones musculares y nerviosas & $\begin{array}{l}\text { - Trauma raquimedular cervical: lesión espinal, lesión diafragmática } \\
\text { - Medicamentos: bloqueadores neuromusculares } \\
\text { - Miastenia gravis } \\
\text { - Esclerosis múltiple } \\
\text { - Distrofia muscular en etapas tardías } \\
\text { - Síndrome de Guillain - Barré }\end{array}$ \\
\hline Obstrucción de la vía aérea superior & $\begin{array}{l}\text { - Hiperplasia amigdalina y adenoidea } \\
\text { - Asma } \\
\text { - Pólipos } \\
\text { - Infecciones: epiglotitis, laringotraquererts } \\
\text { - Traumatismo de la vía aérea superior } \\
\text { - Otras: traqueomalacia, artritis cricoaritenoidea }\end{array}$ \\
\hline Trauma de tórax & $\begin{array}{l}\text { - Fracturas costales } \\
\text { - Tórax inestable } \\
\text { - Hemotórax } \\
\text { - Neumotórax }\end{array}$ \\
\hline
\end{tabular}

Fuente: (Quiroga, Zúñiga, Martínez, \& Mantilla, 2008)

Otro factor importante que pude afectar en el sistema respiratorio es la variabilidad climática del entorno de la persona. La enfermedad respiratoria sigue patrones temporales ya que en las latitudes altas se presenta un incremento de los casos en los meses de invierno, en tanto que en los países tropicales se presenta con mayor frecuencia durante la temporada de lluvias(Correal, Marthá, \& Sarmiento, 2015). 


\section{Enfermedad pulmonar obstructiva crónica (EPOC)}

Vol. 3, núm. 1., (2019)

José Ignacio Macías Alcívar; Jenny Marisela Briones Fajardo; Giannella Lissette Ramírez López; Edwing Xavier Pincay Macías

Una de las enfermedades más recurrentes en la población es la enfermedad pulmonar obstructiva crónica (EPOC). La EPOC representa un problema de salud con importantes implicaciones sociosanitarias, debido a la elevada morbimortalidad que conlleva(Fernández Vargas, y otros, 2001).Se caracteriza esencialmente por una limitación crónica al flujo aéreo poco reversible y asociada principalmente al humo de tabaco(GesEPOC, 2012). Los síntomas que puede presentar el paciente son tos, disnea y la expectoración. En muchas ocasiones, el tratamiento va a conseguir mejorar los síntomas sin cambios apreciables en los valores espirométricos, pero se traducirá en una mejoría en la calidad de vida del enfermo(Cimas Hernando, 2003).

Por lo tanto, esta investigación tiene como objetivo general analizar las características de la enfermedad pulmonar obstructiva crónica (EPOC). La metodología empleada se basó en una investigación documental y bibliográfica a través del análisis de textos, revistas científicas y documentos web.

\section{Método.}

La metodología empleada se basó en investigaciones de tipo documental y bibliográfica donde se puedo analizar las características de la enfermedad pulmonar obstruida crónica a través de sus síntomas, causas, diagnósticos y tratamiento. Aunado a estos tipos de investigación se utilizaron medios como textos, documentos y artículos científicos publicados disponibles en la web. 


\section{Enfermedad pulmonar obstructiva crónica (EPOC)}

Vol. 3, núm. 1., (2019)

José Ignacio Macías Alcívar; Jenny Marisela Briones Fajardo; Giannella Lissette Ramírez López; Edwing Xavier Pincay Macías

\section{Resultados.}

Una de las enfermedades respiratorias más comunes e importantes a nivel mundial es la EPOC. La EPOC abarca aquellas enfermedades respiratorias que cursan con obstrucción no totalmente reversible del flujo aéreo lo cual produce una limitación que es progresiva y que esta asociada a una respuesta inflamatoria (Gené, y otros, 2003).

Es considerada con una enfermedad prevenible y tratable donde en algunas excepciones pueden existir casos significativos de severidad en algunos pacientes. Su componente pulmonar es caracterizado por la limitación del flujo aéreo que no es totalmente reversible y usualmente progresivo. Esta obstrucción se asocia con una respuesta inflamatoria anormal de los pulmones y la vía aérea a partículas nocivas o gases(Félix Hinojosa, 2009).

Por lo cual la definición más exacta y precisa de la EPOC es la aportada por el comité GOLD (Global Initiative in ObstructiveLung).

Enfermedad caracterizada por limitación al flujo aéreo que no es totalmente reversible, usualmente progresiva y asociada a inflamación en los pulmones en respuesta a gases o partículas nocivas. (Molfino, 2004, pág. 445)

Este mismo comité también se encargo de clasificar las distintas EPOC según su gravedad donde busca simplificar el seguimiento, pero no ha sido valorada clínicamente. Esta clasificación se detalla en la Tabla 4. 


\section{Enfermedad pulmonar obstructiva crónica (EPOC)}

Vol. 3, núm. 1., (2019)

José Ignacio Macías Alcívar; Jenny Marisela Briones Fajardo; Giannella Lissette Ramírez López; Edwing Xavier Pincay Macías

\section{Tabla 4. Clasificación de la EPOC según su gravedad}

\begin{tabular}{|c|c|}
\hline Estadio & Caracteristicas \\
\hline $0:$ En riesgo & $\begin{array}{l}\text {-Espirometria normal } \\
\text {-Presencia de sintomas crónicos (tos, } \\
\text { expectoración) }\end{array}$ \\
\hline I: EPOC leve & $\begin{array}{l}\text {-FEV } / \text { FVC }<70 \% \\
\text { - FEV } 1 \geq 80 \% \text { del teórico } \\
\text {-Con o sin presencia de sintomas } \\
\text { crónicos (tos, expectoración) }\end{array}$ \\
\hline \multirow[t]{2}{*}{ II: EPOC moderada } & $\begin{array}{l}\text {-FEV } / \text { FVC }<70 \% \\
\text {-FEV },<\text { del } 80 \% \text { del teórico } y \geq 30 \% \text { del } \\
\text { teórico, con dos subapartados: } \\
\text {-IIA: } \mathrm{FEV}_{1}<80 \% \text { y } \geq 50 \% \text { del teórico } \\
\text {-IIB: } \mathrm{FEV}_{1}<80 \% \text { y } \geq 30 \% \text { del teórico }\end{array}$ \\
\hline & $\begin{array}{l}\text {-Con o sin presencia de sintomas } \\
\text { crónicos (tos, expectoración, disnea) }\end{array}$ \\
\hline III: EPOC grave & $\begin{array}{l}\text {-FEV }, / F V C<70 \% \\
-\mathrm{FEV}_{1}<30 \% \text { del teórico, o FEV1 }<50 \% \\
\text { del teórico con fallo respiratorio } 0 \\
\text { signos de fallo cardiaco derecho. }\end{array}$ \\
\hline
\end{tabular}

FEV; Volumen espiratorio forzado en el primer segundo. FUC: Capacidad vital forzada.

El FEV, se refiere siempre al obtenido tras broncodilatacioin.

Fuente: (Cimas Hernando, 2003)

En la población ecuatoriana que sufre de la EPOC hay una mal nutrición debida al déficit de los proteicos. Esto empeorasu pronóstico por lo que es importante el diseño de unprograma de educación nutricional que incluya elaporte de 1.2 a $1.5 \mathrm{Kg} /$ día de proteínas, así comola disminución de los carbohidratos, en la dieta(Orozco \& Orozco, 2010).

La formación de esta enfermedad es variada y va a depender de la calidad de vida que tiene el paciente. Entre sus factores de riesgo se encuentran hábitos personales y exposición a 


\section{Enfermedad pulmonar obstructiva crónica (EPOC)}

Vol. 3, núm. 1., (2019)

José Ignacio Macías Alcívar; Jenny Marisela Briones Fajardo; Giannella Lissette Ramírez López; Edwing Xavier Pincay Macías

contaminantes en el domicilio y laborales, pero de todos ellos, el mayor es el tabaquismo(Amigo, Erazo, Oyarzún, Bello, \& Peruga, 2006).

En el Ecuador los factores de riesgo también dependen del sexo del paciente y de las labores que realicen en el día a día. En mujeres, el factor más común es cocinar con leña y carbón; por lo que se debe educar a la población de escasos recursos económicos sobre la acción dañina del uso de estos materiales en la cocina de las viviendas rurales y periurbanas(Orozco \& Orozco, 2010).

Estar en presencia de los humos del cigarrillo y de la leña altera los organismos que conforman el sistema respiratorio.

Se ha informado que existe activación de macrófagos y de células epiteliales por los productos tóxicos del cigarro, humo de leña o de irritantes inespecíficos.Esta activación, a su vez, provoca la liberación de diferentes mediadores inflamatorios que promueven la quimiotaxis y la activación de linfocitos $\mathrm{T}$ y otras células polimorfonucleares (CPMN) amplificando así, el proceso inflamatorio.(Sansores, y otros, 2012, pág. 17)

Es por ello, que esta enfermedad puede afectar la calidad de vida de los pacientes que la sufren por lo que se ven alterados tantos su aspecto físico como psicológico.

Desde la fase inicial aguda, en la que los pacientes sufren un período de crisis caracterizado por un desequilibrio físico, social y psicológico, acompañado de ansiedad, miedo y desorientación, hasta la asunción del carácter crónico del trastorno, que implica, en mayor o menor medida, cambios permanentes en la actividad física, laboral y social de los 


\section{Enfermedad pulmonar obstructiva crónica (EPOC)}

Vol. 3, núm. 1., (2019)

José Ignacio Macías Alcívar; Jenny Marisela Briones Fajardo; Giannella Lissette Ramírez López; Edwing Xavier Pincay Macías

pacientes, condición que suele durar toda la vida.(Vinaccia, Quiceno, Zapata, Obesso, \& Quintero, 2011, pág. 93)

Es decir que estos pacientes de EPOC sufren de niveles de ansiedad y depresión acompañadas de la descomposición del cuadro médico de la enfermedad por lo que es necesario una estrategia de afrontamiento.

Existen síntomas que puedan determinar un diagnostico subjetivo. Los síntomas principales son la disnea, la tos y la expectoración(GesEPOC, 2012). La disnea es la causa más importante de disconfort, ansiedad y disminución de la calidad de vida, la cual se describe como un incremento del esfuerzo para respirar, necesidad de aire, falta de aire en los pulmones o respiración dificultosa (“a bocanadas”)(Cimas Hernando, 2003).

La tos es considerada como el primer síntoma de pacientes que sufren EPOC por lo que el paciente tiende a ignorarlo solo hasta que se vuelve progresiva. En algunos casos de EPOC la tos puede ser improductiva, y en otras ocasiones, los pacientes pueden desarrollar limitación al flujo aéreo sin que aparezca la tos(Cimas Hernando, 2003).

La expectoración es el material que se expulsa por la boca, procedente del tracto respiratorio inferior, por medio de la tos(Galera, Carrera, \& Ortega, 2010). La tos con expectación se produce por la producción de moco por el consumo de tabaco. Esto es debido al aumento de las glándulas caliciformes y submucosas del árbol bronquial, aunado a esto la composición del esputo es diferente en pacientes de EPOC que, en sujetos sanos, modificándose la acción de aclaramiento de la vía aérea(Cimas Hernando, 2003). 


\section{Enfermedad pulmonar obstructiva crónica (EPOC)}

Vol. 3, núm. 1., (2019)

José Ignacio Macías Alcívar; Jenny Marisela Briones Fajardo; Giannella Lissette Ramírez López; Edwing Xavier Pincay Macías

Ahora es necesario seguir un patrón clínico si se tiene sospecha de padecer EPOC. Debe considerarse en todas las personas adultas con exposición a factores de riesgo, básicamente el habito tabáquico (con una exposición de al menos 10 años-paquete) que presentan tos crónica, con o sin producción de esputo o disnea(GesEPOC, 2012). Por lo cual se deben seguir unos pasos establecidos para el diagnóstico de EPOC en un paciente tal cual se observa en la Figura 6.

Poder determinar una evaluación diagnostica con exactitud recae en evaluar los signos a través de un examen físico como estudio inicial. Este estudio puede revelar un aumento del tiempo de la espiración forzada (mayor de 5 segundos) o sibilancias audibles solo durante dicha maniobra, como también la disminución de la excursión diafragmática y la extracción de la pared costal inferior durante la inspiración (Gené, y otros, 2003).

Una de las primeras técnicas diagnósticas es la espirometría, la cual es la prueba más fiable para la evaluación de la respiración. Mide la cantidad de aire de manera forzada en función del tiempo, lo que depende del calibre de los bronquios, de las propiedades elásticas del tórax y de los pulmones, así como de la integridad de los músculos respiratorios(Vargas-Domínguez, y otros, 2011).De los diversos índices derivados de una espiración forzada, el VEF y la CVF son los más usados(Gutiérrez, y otros, 2007). 


\section{Enfermedad pulmonar obstructiva crónica (EPOC)}

Vol. 3, núm. 1., (2019)

José Ignacio Macías Alcívar; Jenny Marisela Briones Fajardo; Giannella Lissette Ramírez López; Edwing Xavier Pincay Macías

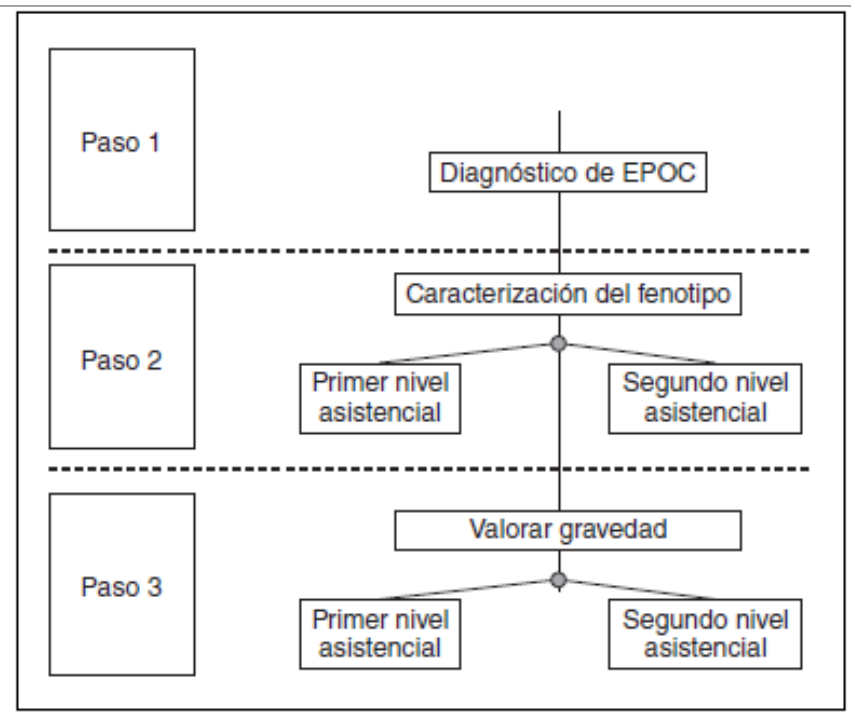

Figura 6. Pasos para la evaluación diagnostica de paciente con EPOC

Fuente: (GesEPOC, 2012)

Esto procede a un algoritmo que se debe realizar los pacientes cuando se sospecha de que sufre EPOC. La sospecha clínica debe confirmarse por medio de una espirometría forzada con prueba broncodilatadora realizada en la fase estable de la enfermedad, imprescindible para establecer el diagnóstico de EPOC(GesEPOC, 2012). Este se puede observar en la Figura 7. 


\section{Enfermedad pulmonar obstructiva crónica (EPOC)}

Vol. 3, núm. 1., (2019)

José Ignacio Macías Alcívar; Jenny Marisela Briones Fajardo; Giannella Lissette Ramírez

López; Edwing Xavier Pincay Macías

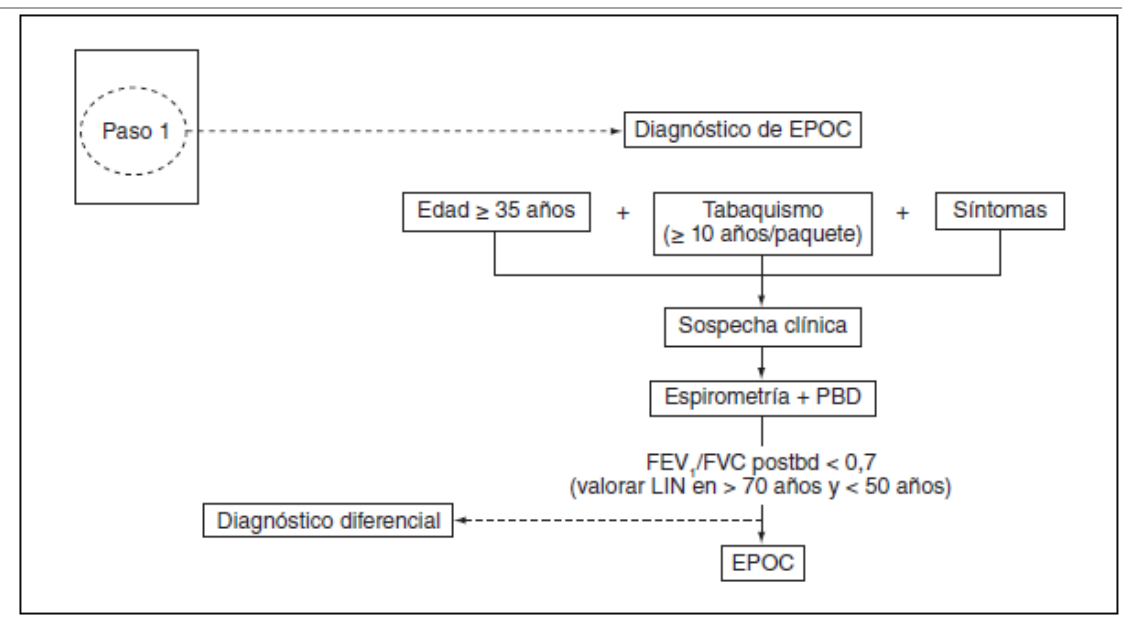

Figura 7. Primer paso en la evaluación del paciente con EPOC. Algoritmo de sospecha diagnostica y diagnóstico de la EPOC. LIN: límite inferior de la normalidad; PBD: prueba broncodilatadora.

Fuente: (GesEPOC, 2012)

Seguidamente se pueden realizar técnicas o pruebas que puedan determinar con mayor precisión la enfermedad en el paciente. En la Tabla 5 se muestra las exploraciones complementarias útiles en el diagnóstico de la EPOC. 


\section{Enfermedad pulmonar obstructiva crónica (EPOC)}

Vol. 3, núm. 1., (2019)

José Ignacio Macías Alcívar; Jenny Marisela Briones Fajardo; Giannella Lissette Ramírez López; Edwing Xavier Pincay Macías

Tabla 5. Exploraciones complementarias útiles en el diagnóstico de la EPOC

\begin{tabular}{l}
\hline Pruebas diagnósticas iniciales \\
\hline Espirometría forzada \\
\hline Prueba broncodilatadora \\
\hline Radiografía de tórax \\
\hline Pruebas diagnósticas adicionales \\
\hline Gasometría arterial \\
\hline Pulsioximetría \\
\hline Análisis de sangre y orina \\
\hline Electrocardiograma \\
\hline Determinación de alfa-1-antitripsina sérica \\
\hline Pruebas diagnósticas opcionales \\
\hline Test de difusión \\
\hline Pletismografía \\
\hline Determinación de la distensibilidad pulmonar \\
\hline Test de la marcha de 6012 minutos \\
\hline Ergometría respiratoria \\
\hline Oximetría nocturna \\
\hline Polisomnografía \\
\hline Tomografía computarizada torácica \\
\hline Ecocardiograma \\
\hline Hemodinámica pulmonar \\
\hline
\end{tabular}

Fuente: (Walther, y otros, 2001)

Para contrarrestar este efecto negativo de las enfermedades se encuentra una de las defensas que tiene el organismo como lo son las plaquetas. Estas intervienen de manera activa en diversos procesos fisiológicos y patológicos vinculados con la inflamación, la remodelación tisular y la defensa innata contra microorganismos(Guzmán Grenfell, Maldonado Noriega, Mendoza Atencio, \& Hicks Gómez, 2005). 


\section{Enfermedad pulmonar obstructiva crónica (EPOC)}

Vol. 3, núm. 1., (2019)

José Ignacio Macías Alcívar; Jenny Marisela Briones Fajardo; Giannella Lissette Ramírez López; Edwing Xavier Pincay Macías

También se pueden aplicar una serie d tratamientos que ayudarna a la mejora en salud del paciente y a un aumento en la calidad de vida del mismo y sus familiares. Por lo cual, los objetivos del tratamiento de la EPOC se centran en mejorar los síntomas y en aumentar la supervivencia(Walther, y otros, 2001). Los pilares básicos del tratamiento se pueden observar en la Tabla 6. Posteriormente, se realizan una serie de tratamientos para pacientes estables las cuales se detallan en la Figura 8.

Tabla 6. Objeticos del tratamiento de la EPOC

\begin{tabular}{l}
\hline Abandonar el hábito tabáquico \\
\hline Aliviar los síntomas y prevenir las agudizaciones \\
\hline Mejorar la calidad de vida y la tolerancia al ejercicio \\
\hline Preservar la función pulmonar o reducir su deterioro \\
\hline Aumentar la supervivencia \\
\hline Prevenir, detectar y tratar precozmente las complicaciones \\
\hline Minimizar los efectos adversos de la medicación
\end{tabular}

Fuente: (Walther, y otros, 2001) 


\section{Enfermedad pulmonar obstructiva crónica (EPOC)}

Vol. 3, núm. 1., (2019)

José Ignacio Macías Alcívar; Jenny Marisela Briones Fajardo; Giannella Lissette Ramírez López; Edwing Xavier Pincay Macías

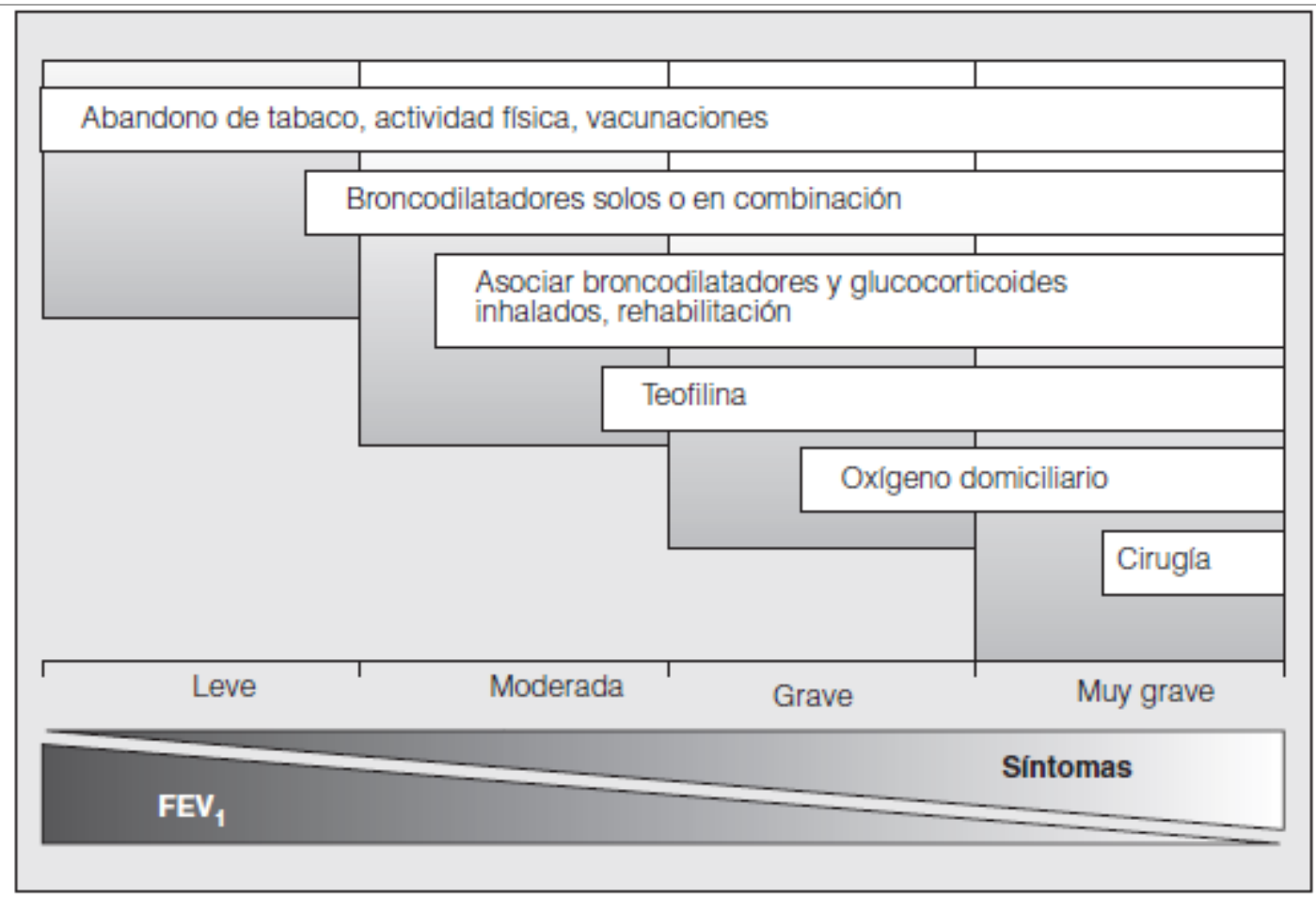

Figura 8. Tratamiento de la EPOC en fase estable. FEV $V_{1}$ volumen espiratorio forzado en

\section{el primer segundo}

Fuente: (Peces-Barba, y otros, 2008)

El abandono del tabaco, actividad física y vacunaciones pertenecen a los tratamientos no farmacológicos. Antes de realizar cualquiera de estos tratamientos no medicados es necesario aplicar un sistema educativo para el paciente. Esta educación debe comenzar desde la primera visita la cual debe incluir a algún familiar y estará formando parte del método de rehabilitación que incluye material escrito, reuniones grupales, psicólogos y kinesiólogo especializados (Gené, y otros, 2003). Los programas educativos para promover el autocuidado se han dirigido a la deshabituación tabáquica, mejorar el ejercicio, nutrición, autotratamiento de las agudizaciones, técnicas de inhalación o adaptación a las actividades de la vida diaria(Escarrabill, 2003). 


\section{Enfermedad pulmonar obstructiva crónica (EPOC)}

Vol. 3, núm. 1., (2019)

José Ignacio Macías Alcívar; Jenny Marisela Briones Fajardo; Giannella Lissette Ramírez López; Edwing Xavier Pincay Macías

Dentro de los aspectos psicológicos, y que representa un papel importante en la educación del paciente para enfrentar la enfermedad es el control de la ansiedad y la depresión. Estas enfermedades colaterales tienen una gran influencia en los marcadores de calidad de vida y pueden ser difíciles de diagnosticar, una consulta con psiquiatría es necesaria para que se realice una anamnesia psiquiátrica estructurada y se implemente tratamiento específico(Molfino, 2004).

El otro aspecto es dejar el habito de fumar y se puede lograr explicando las causas que producen la enfermedad, pero el tabaquismo es una adicción crónica. Mientras que una minoría consigue la abstinencia definitiva después del primer intento de abandono, la mayoría de los fumadores persisten con períodos de remisión y recaída hasta que consiguen recuperarse definitivamente(Sansores, y otros, 2012). Estas recaídas son parte del tratamiento psicológico y del autocontrol que debe llevar el paciente.

Otros de los tratamientos no farmacológicos es la aplicación de alguna actividad fpisica o de la permitida en la rehabilitación según sea el caso. El entrenamiento permite estimular la función cardiaca y pulmonar que permita una utilización más eficiente del oxígeno a nivel periférico(Zenteno, y otros, 2007). Esta estimulación permite una mejor respiración y por ende una mejor condición del sistema respiratorio.

Las vacunas que se consideran mandatarias son la vacuna antineumocócica tanto polisacárido o conjugada por lo menos una dosis cada 3-5 años y la vacuna antigripal en forma anual. Los pacientes con EPOC tienen un alto riesgo de hospitalización por influenza y la vacuna reduce esta complicación hasta en un 39\%. Los beneficios de la vacuna 


\section{Enfermedad pulmonar obstructiva crónica (EPOC)}

Vol. 3, núm. 1., (2019)

José Ignacio Macías Alcívar; Jenny Marisela Briones Fajardo; Giannella Lissette Ramírez López; Edwing Xavier Pincay Macías

antineumocócica están menos establecidos, pero datos preliminares demuestran una eficiencia del 65\%.(Molfino, 2004, pág. 451)

Ahora los tratamientos farmacológicos van a depender primero del medicamento reemplazante del tabaquismo y luego un tratamiento de broncodilatador y esteroides inhaladas. Estos se pueden visualizar en la Tabla 7 y 8 respectivamente.

Tabla 7. Medicamentos para el tratamiento del tabaquismo

\begin{tabular}{|c|c|c|c|c|c|}
\hline & \multicolumn{2}{|c|}{ No nicotínicos } & \multicolumn{3}{|c|}{ Nicotínicos } \\
\hline & Varenicline & $\begin{array}{l}\text { Anfebutamona } \\
\text { (Bupropión) }\end{array}$ & Parche & Goma de mascar & Inhalador \\
\hline $\begin{array}{l}\text { Mecanismo } \\
\text { de acción }\end{array}$ & $\begin{array}{l}\text {-Agonista parcial } \\
\text { de receptores } \alpha 4 \beta_{2}\end{array}$ & $\begin{array}{l}\text {-Mecanismo de } \\
\text { acción poco } \\
\text { conocido. } \\
\text { Aumenta la } \\
\text { concentración } \\
\text { de dopamina en } \\
\text { el cerebro. Actúa } \\
\text { en locus coeruleus }\end{array}$ & $\begin{array}{l}\text {-Actúa a nivel de } \\
\text { receptores } \\
\text { nicotínicos del } \\
\text { SNC }\end{array}$ & $\begin{array}{l}\text {-Actúa a nivel de } \\
\text { receptores } \\
\text { nicotínicos } \\
\text { del SNC }\end{array}$ & $\begin{array}{l}\text {-Actúa a nivel de } \\
\text { receptores } \\
\text { nicotínicos del SNC }\end{array}$ \\
\hline $\begin{array}{l}\text { Dosis y tiempo } \\
\text { de } \\
\text { administración }\end{array}$ & $\begin{array}{l}\text {-0.5 mg al día } \\
\text { durante tres dias. } \\
\text { iniciando una } \\
\text { semana antes de } \\
\text { suspender el } \\
\text { tabaco; continuar } \\
\text { con } 0.5 \mathrm{mg} \text { dos } \\
\text { veces al dia durante } \\
\text { cuatro dias; a } \\
\text { continuación. } 1 \mathrm{mg} \\
\text { dos veces al dia } \\
\text { durante tres meses } \\
\text { Instruir para dejar de } \\
\text { fumar el día ocho. } \\
\text { cuando la dosis } \\
\text { aumenta a } 1 \mathrm{mg} \\
\text { dos veces al dia }\end{array}$ & $\begin{array}{l}-150 \mathrm{mg} \text { al día } \\
\text { durante seis dias, } \\
\text { una semana antes } \\
\text { de suspender el } \\
\text { consumo de } \\
\text { tabaco; seguir con } \\
150 \mathrm{mg} \text { dos veces } \\
\text { al dia durante ocho } \\
\text { a nueve semanas }\end{array}$ & $\begin{array}{l}\text {-Existen } 2 \\
\text { presentaciones: } \\
1 . \\
\text { Etapa } 1.21 \\
\text { mg } \times 4 \text { semanas } \\
\text { Etapa } 2.14 \\
\text { mg } \times 2 \text { semanas } \\
\text { Etapa } 3.7 \\
\mathrm{mg} \times 2 \text { semanas } \\
2 . \\
15 \mathrm{mg} / 16 \mathrm{~h} \\
12 \mathrm{semanas} \\
10 \mathrm{mg} / 16 \mathrm{~h} \\
2 \mathrm{a} 3 \text { semanas } \\
5 \mathrm{mg} / 16 \mathrm{~h} \\
2 \mathrm{a} 3 \text { semanas }\end{array}$ & $\begin{array}{l}\text {-Presentación } \\
4 \text { y } 2 \text { mg } \\
20 \text { piezas al día } \\
\text { (4 mg) por } 8-12 \\
\text { semanas }\end{array}$ & $\begin{array}{l}\text {-Presentación en } \\
\text { cartucho de } 10 \mathrm{mg} \\
\text { Fase } 1.10 \text { a } 20 \\
\text { mg/dia (semana } \\
1 \text { a la } 6 \text { ) } \\
\text { Fase } 2.10 \mathrm{mg} / 2 \text { días, } \\
\text { (semana } 7 \text { a } 10 \text { ) } \\
\text { Fase } 3.10 \mathrm{mg} / \text { cada } 3 \\
\text { dias (semana } 10 \text { a } \\
\text { la 12) }\end{array}$ \\
\hline Efecto & $\begin{array}{l}\text {-Liberación de } \\
\text { dopamina en SNC } \\
40 \% \text { menor en } \\
\text { comparación con } \\
\text { nicotina }\end{array}$ & $\begin{array}{l}\text {-Antidepresivo y } \\
\text { disminuye los } \\
\text { síntomas de } \\
\text { abstinencia }\end{array}$ & $\begin{array}{l}\text {-Disminuye los } \\
\text { síntomas de } \\
\text { abstinencia }\end{array}$ & $\begin{array}{l}\text {-Disminuye los } \\
\text { síntomas de } \\
\text { abstinencia }\end{array}$ & $\begin{array}{l}\text {-Disminuye los } \\
\text { síntomas de } \\
\text { abstinencia }\end{array}$ \\
\hline $\begin{array}{l}\text { Reacciones } \\
\text { secundarias }\end{array}$ & $\begin{array}{l}\text {-Náusea en el } 30 \% \\
\text { de los pacientes. } \\
\text { tolerable, disminuye } \\
\text { después de } 3 \\
\text { semanas de } \\
\text { tratamiento. } \\
\text { problemas de } \\
\text { sueño, sueños } \\
\text { vividos o anormales }\end{array}$ & $\begin{array}{l}\text { - Insomnio, sequedad } \\
\text { de boca, crisis } \\
\text { convulsivas (menos } \\
\text { de } 0.1 \% \text { de casos). } \\
\text { reacciones } \\
\text { cutáneas, mareo }\end{array}$ & $\begin{array}{l}\text {-Cefalea, irritación } \\
\text { cutánea, insomnio. } \\
\text { mareo, mialgias }\end{array}$ & $\begin{array}{l}\text {-Gastritis, dolor } \\
\text { articular } \\
\text { temporomaxilar, } \\
\text { hipo, mal sabor } \\
\text { de boca }\end{array}$ & $\begin{array}{l}\text {-Irritación de la } \\
\text { garganta, tos, rinitis, } \\
\text { cefalea, náuseas }\end{array}$ \\
\hline Contraindicado & $\begin{array}{l}\text {-Alergia al principio } \\
\text { activo. Usar con } \\
\text { precaución en } \\
\text { pacientes con } \\
\text { historia de trastorno } \\
\text { depresivo o trastornos } \\
\text { psiquiátricos }\end{array}$ & $\begin{array}{l}\text {-Historia de crisis } \\
\text { convulsivas } \\
\text { Traumatismo } \\
\text { craneoencefálico. } \\
\text { Anorexia y/o } \\
\text { bulimia } \\
\text { Uso simultáneo } \\
\text { de fármacos } \\
\text { inhibidores de la } \\
\text { monoamino-oxidasa }\end{array}$ & $\begin{array}{l}\text {-Infarto al miocardio } \\
\text { un mes antes } \\
\text { Hipertensión } \\
\text { arterial no controlada } \\
\text { Cardiopatia no } \\
\text { controlada } \\
\text { Arritmia cardiaca } \\
\text { no controlada } \\
\text { Uso cuidadoso } \\
\text { durante el embarazo }\end{array}$ & $\begin{array}{l}\text {-Infarto al miocardio } \\
\text { un mes antes } \\
\text { Artropatia } \\
\text { temporomaxilar } \\
\text { Inflamación } \\
\text { orofaríngea } \\
\text { Trastornos } \\
\text { dentarios }\end{array}$ & $\begin{array}{l}\text {-Infarto al miocardio } \\
\text { un mes antes }\end{array}$ \\
\hline Beneficios & $\begin{array}{l}\text {-Duplica la } \\
\text { probabilidad de } \\
\text { abstinencia a } 12 \\
\text { meses versus } \\
\text { bupropión. } \\
\text { cuadruplica la } \\
\text { probabilidad de } \\
\text { abstinencia a } 12 \\
\text { meses versus } \\
\text { placebo' }\end{array}$ & $\begin{array}{l}\text { - Doble acción. } \\
\text { Antidepresivo y } \\
\text { disminuye síntomas } \\
\text { de abstinencia } \\
\text { Menor incremento } \\
\text { de peso una vez } \\
\text { que ha dejado } \\
\text { de fumar }\end{array}$ & $\begin{array}{l}\text {-Fácil } \\
\text { administración } \\
\text { Se invierte poco } \\
\text { tiempo en } \\
\text { explicarle al } \\
\text { paciente la } \\
\text { forma de } \\
\text { administración }\end{array}$ & $\begin{array}{l}\text {-Preferencia del } \\
\text { paciente }\end{array}$ & $\begin{array}{l}\text {-Fácil administración. } \\
\text { disponibilidad y } \\
\text { preferencia del } \\
\text { paciente }\end{array}$ \\
\hline
\end{tabular}

Fuente: (Sansores, y otros, 2012) 


\section{Enfermedad pulmonar obstructiva crónica (EPOC)}

Vol. 3, núm. 1., (2019)

José Ignacio Macías Alcívar; Jenny Marisela Briones Fajardo; Giannella Lissette Ramírez López; Edwing Xavier Pincay Macías

\section{Tabla 8. Dosis y posología del broncodilatador y esteroides inhalados}

\begin{tabular}{|c|c|c|c|}
\hline Medicamento & $\begin{array}{l}\text { Dosis medida por } \\
\text { Inhalación (mcg) }\end{array}$ & $\begin{array}{l}\text { Nebulizado } \\
\text { (mg) }\end{array}$ & $\begin{array}{l}\text { Duración de } \\
\text { acción }(\mathrm{h})\end{array}$ \\
\hline \multicolumn{4}{|l|}{ Anticolinérgicos de acción corta } \\
\hline Bromuro de Ipratropio & $40-80$ & $0.25-0.5$ & $6-8$ \\
\hline \multicolumn{4}{|l|}{ Anticolinérgicos de acción prolongada } \\
\hline Bromuro de Tiotropio & 18 & - & 24 \\
\hline \multicolumn{4}{|l|}{$\beta_{2}$-agonistas de acción corta } \\
\hline Salbutamol & $100-200^{x}$ & $2.5-5.0$ & $4-6$ \\
\hline Terbutalina & 250 & $5-10$ & $4-6$ \\
\hline \multicolumn{4}{|l|}{$\beta_{2}$-agonistas de acción prolongada } \\
\hline Formoterol & 12 & - & 12 \\
\hline Salmeterol & 50 & - & 12 \\
\hline Indacaterol & $150-300$ & - & 24 \\
\hline \multicolumn{4}{|c|}{$\begin{array}{l}\text { Combinación de } \beta_{2} \text {-agonista + anticolinérgico } \\
\text { de acción corta }\end{array}$} \\
\hline (Salbutamol + Anticolinérgico) IDM & $90 \mathrm{mg} / 20 \mathrm{mg}$ & $2.5 / 0.5$ & $4-6$ \\
\hline Administrado con Respimat & $100 \mathrm{mg} / 20 \mathrm{mg}$ & & $4-6$ \\
\hline \multicolumn{4}{|l|}{ Esteroides inhalados } \\
\hline Budesonida IPS Turbohaler & $100-200$ & $0.125-0.25$ & 6 \\
\hline Fluticasona IDM & $50-250$ & $0.5-2$ & 6 \\
\hline Mometasona IPS Twisthaler & 200 & & \\
\hline Ciclosenida IDM & $50,100,200$ & & \\
\hline \multicolumn{4}{|c|}{$\begin{array}{l}\text { Combinación de broncodilatador y esteroide } \\
\text { (en un mismo dispositivo) }\end{array}$} \\
\hline Formoterol + Budesonida (IPS Turbohaler) & $4.5 / 804.5 / 1609 / 320$ & - & 12 \\
\hline Salmeterol + Fluticasona (IDM) & $25 / 50-25 / 125-25 / 250$ & - & 12 \\
\hline Salmeterol + Fluticasona (IPS Diskus) & $50 / 100-50 / 250-50 / 500$ & - & 12 \\
\hline Formoterol + Mometasona (IDM) & $5 / 1005 / 200$ & - & 12 \\
\hline \multicolumn{4}{|c|}{$\begin{array}{l}\text { Combinación de broncodilatador y esteroide } \\
\text { (en dispositivos independientes) }\end{array}$} \\
\hline Formoterol + El & Ver arriba & - & 12 \\
\hline Salmeterol + El & & - & 12 \\
\hline Indacaterol + El & & - & 12 \\
\hline Tiotropio +El & & - & 12 \\
\hline \multicolumn{4}{|l|}{ Inhibidores de la fosfodiestereasa $\mathbf{4}$} \\
\hline Roflumilast & $500 \mathrm{mg}$ vía oral & & 24 \\
\hline
\end{tabular}

Fuente: (Sansores, y otros, 2012)

Una de los tratamientos alternos que derivan de culturas de la sociedad son las plantas medicinales. 


\section{Enfermedad pulmonar obstructiva crónica (EPOC)}

Vol. 3, núm. 1., (2019)

José Ignacio Macías Alcívar; Jenny Marisela Briones Fajardo; Giannella Lissette Ramírez López; Edwing Xavier Pincay Macías

Varias plantas medicinales son utilizadas en sistemas tradicionales de salud para el tratamiento de enfermedades respiratorias, incluso algunas de ellas han sido investigadas para verificar su eficacia. Las especies más usadas pertenecieron a la familia Asteraceae(15 especies, 16,67\%), seguido por Lamiaceae y Fabaceae (8,89\% y 5,56\%). En los preparados para problemas respiratorios se utilizan con más frecuencia hojas de plantas, seguido de la planta entera, flores y tallos(Bussmann, 2010, pág. 331).

Por último, están los tratamientos quirúrgicos. El tratamiento quirúrgico, a través de la cirugía de reducción de volumen ha mejorado los síntomas clínicos con mayor tolerancia al esfuerzo, mejoría de la disnea alcanzando una supervivencia de más de 2 años sin deterioro clínico ni funcional(Páez Prats, 1999).

\section{Discusión y conclusiones.}

El sistema respiratorio es fundamental para el equilibrio bioquímico, físico y psicológico de las personas. La importancia radica en que la respiración juega un papel importante en el desarrollo de las actividades cotidianas del ser humano ya que permiten una mayor habilidad y destreza física del organismo. El intercambio de gases con la atmosfera permite una mejor circulación de la sangre aunado a que el oxigeno que entra es transformado por dióxido de carbono que es expulsado por el metabolismo celular de este sistema. No solo inhala oxígeno, sino que también inhala otros gases donde estos últimos, en condiciones establos del organismo, puede transformar y expulsarlo al medio ambiente. Es por ello que recae en importancia el significado del aparto respiratorio y la funcionabilidad que tienen cada miembro en desarrollar esta tarea. Este sistema respiratorio también cuenta con un proceso evolutivo que empieza en la 


\section{Enfermedad pulmonar obstructiva crónica (EPOC)}

Vol. 3, núm. 1., (2019)

José Ignacio Macías Alcívar; Jenny Marisela Briones Fajardo; Giannella Lissette Ramírez López; Edwing Xavier Pincay Macías

etapa embrionaria desde la gestación hasta la etapa alveolar que dura hasta los 3 años de vida aproximadamente.

Es por ello que tener un óptimo sistema respiratorio mejora la calidad de vida del individuo pero cuando se rompe este equilibrio y empieza a aparecer deformaciones y circunstancia que alteran el funcionamiento de los diferentes órganos que los conforman entonces se está hablando de padecimiento de alguna enfermedad respiratoria. Esta enfermedad respiratoria se fundamenta en que el sistema respiratorio ya no realiza eficazmente el proceso químico y físico de la transformación del oxígeno. Este padecimiento se refleja a través de la insuficiencia respiratoria. Esta insuficiencia en la respiración está relacionada con parámetros como la calidad de vida sociocultural y económica del individuo y el consumo de tabaco. Otro aspecto fundamental para el desarrollo de estas enfermedades son las condiciones laborales y ambientales a las que se ve involucrado la persona durante períodos de tiempos considerados.

Una de estas enfermedades es la EPOC la cual se caracteriza por una deficiencia en el flujo aéreo que se vuelve poco reversible y que se manifiesta a través de tos, disnea y expectación. Las causas principales de esta enfermedad son la inhalación de humo de leña y de tabaco. Los diagnósticos establecidos están determinados principalmente por inspección física del paciente para luego aplicar espirometría como método principal de detección, seguidamente de métodos secundarios como radiografías TAC, electrocardiograma, ecocardiograma, entre otros. El tratamiento va depender de varios factores como tratamientos no farmacológicos como planes educativos, método de dejar el cigarrillo, autocontrol, ayudas psicológicas, actividad física y vacunas. También están los tratamientos farmacológicos que empiezan con medicamentos que ayudan a sustituir al tabaco para luego realizar tratamiento según sea la 


\section{Enfermedad pulmonar obstructiva crónica (EPOC)}

Vol. 3, núm. 1., (2019)

José Ignacio Macías Alcívar; Jenny Marisela Briones Fajardo; Giannella Lissette Ramírez López; Edwing Xavier Pincay Macías

gravedad. La aplicación de estos diagnósticos y tratamientos puede mejorar la calidad de vida del paciente como también de las personas que lo rodean.

\section{Referencias.}

Abú Shams, K., Boldú, J., Tiberio, G., Tabar, A., Fernández Infante, B., \& Labarta, N. (2005). Registro de enfermedades respiratorias de origen laboral en Navarra. In Anales del Sistema Sanitario de Navarra (Vol. 28). Gobierno de Navarra. Departamento de Salud, 135-143.

Amigo, H., Erazo, M., Oyarzún, M., Bello, S., \& Peruga, A. (2006). Tabaquismo y enfermedad pulmonar obstructiva crónica: determinación de fracciones atribuibles. Revista médica de Chile, 134(10), 1275-1282.

Asenjo, C. A. (2017). Características anátomo-funcional del aparato respiratorio durante la infancia. Revista Médica Clínica Las Condes, 28(1), 7-19.

Bussmann, R. W. (2010). Plantas medicinales utilizadas en Perú para el tratamiento de enfermedades respiratorias. Revista peruana de Biología, 17(3), 331-346.

Cimas Hernando, J. E. (2003). Importancia de los síntomas en la EPOC. Medifam, 13(3), 46-55.

Correal, M. E., Marthá, J. E., \& Sarmiento, R. (2015). Influencia de la variabilidad climática en las enfermedades respiratorias agudas en Bogotá. Biomédica, 35(2)., 130-138.

Díaz, M. M., Silva, A. M., Pérez, D. L., Muguercia, H. L., Torres, M. L., Assef, H. P., \& Arias, E. R. (2003). Nutrición y función respiratoria. Acta medica, 11(1), 26-37.

Escarrabill, J. (2003). Enfermedad pulmonar obstructiva crónica (EPOC): visión global y continuidad de cuidados. In Anales de Medicina Interna (Vol. 20, No. 7). Arán Ediciones, SL., 5-7.

Félix Hinojosa, E. C. (2009). Enfermedad pulmonar obstructiva crónica (EPOC). Acta médica peruana, 26(4), 188-191.

Fernández Vargas, A. M., Bujalance Zafra, M. J., Leiva Fernández, F., Martos Crespo, F., García Ruíz, A. J., de la Cuesta, S., \& Alarcón, F. (2001). Salud autopercibida, apoyo social y familiar de los pacientes con enfermedad pulmonar obstructiva crónica. Medifam, 11(9), 44-53. 


\section{Enfermedad pulmonar obstructiva crónica (EPOC)}

Vol. 3, núm. 1., (2019)

José Ignacio Macías Alcívar; Jenny Marisela Briones Fajardo; Giannella Lissette Ramírez

López; Edwing Xavier Pincay Macías

Galera, R., Carrera, L. G., \& Ortega, B. (2010). Enfermedades del aparato respiratorio. Medicine-Programa de Formación Médica Continuada Acreditado, 10(63), 4323-4331.

Gené, R. J., Giugno, E., Abate, E., Figueroa Casas, J. C., Mazzei, J. A., \& Schiavi, E. (2003). Nuevo consenso argentino de la enfermedad pulmonar obstructiva crónica. Medicina (Buenos Aires), 63(5), 419-446.

GesEPOC. (2012). Guía de Práctica Clínica para el diagnóstico y tratamiento de pacientes con Enfermedad Pulmonar Obstructiva Crónica (EPOC)-Guía Española de la EPOC (GesEPOC). Archivos de Bronconeumología, 48, 2-58.

Gutiérrez, M., BeroÍza, T., Borzone, G., Caviedes, I., Céspedes, J., Gutiérrez, M., \& ... Schonffeldt, P. (2007). Espirometría: Manual de procedimientos. Sociedad Chilena de Enfermedades Respiratorias, 2006. Revista chilena de enfermedades respiratorias, 23(1), $31-42$.

Guzmán Grenfell, A. M., Maldonado Noriega, L., Mendoza Atencio, R., \& Hicks Gómez, J. J. (2005). La función plaquetaria más allá de la hemostasis: participación en las enfermedades respiratorias. Revista del Instituto Nacional de Enfermedades Respiratorias, 18(3), 240-246.

López, A. (2006). Patología del sistema respiratorio. Slano, Canadá: Atlantic Veterinary College, 1-18.

Molfino, N. A. (2004). Tratamiento de pacientes con enfermedad pulmonar obstructiva crónica (EPOC) por médicos clínicos generales. Medicina (Buenos Aires), 64(5), 445-454.

Orozco, S. A., \& Orozco, J. C. (2010). Prevalencia, factores de riesgo, y estado nutricional de pacientes con enfermedad pulmonar obstructiva crónica, en el hospital Guayaquil, 2008. Medicina, 16(1), 43-49.

Páez Prats, A. (1999). Enfermedad pulmonar obstructiva crónica: Un alerta en las postrimerías del siglo. Revista Cubana de Medicina, 38(2), 95-97.

Peces-Barba, G., Barberà, J. A., Agustí, A. C., Casas, A., Izquierdo, J. L., \& ... Viejo, J. L. (2008). Guía clínica SEPAR-ALAT de diagnóstico y tratamiento de la EPOC. Arch Bronconeumol, 44(5), 271-281.

Quiroga, I. C., Zúñiga, E. C., Martínez, S. X., \& Mantilla, J. M. (2008). Diagnóstico y manejo de la insuficiencia respiratoria aguda. NCT Neumología y Cirugía de Tórax, 67(1), 24-33. 


\section{Enfermedad pulmonar obstructiva crónica (EPOC)}

Vol. 3, núm. 1., (2019)

José Ignacio Macías Alcívar; Jenny Marisela Briones Fajardo; Giannella Lissette Ramírez López; Edwing Xavier Pincay Macías

Reiriz Palacios, J. (28 de Abril de 2019). SISTEMA RESPIRATORIO: ANATOMÍA. Obtenido de Informe Virtual. Escuela Universitaria de Enfermería. Universidad de Barcelona: https://www.infermeravirtual.com/files/media/file/97/Sistema\%20respiratorio.pdf?13586 05945

Sánchez, I. (2001). Desarrollo del aparato respiratorio y diferencias anatomofuncionales entre el lactante y el adulto. Pediatría al día, 17, 251-254.

Sansores, R. H., Kaldman, M. A., Argote-Greene, L. M., Flores, E. V., Suárez, C. C., Angulo, S. C., \& ... Trujillo, F. F. (2012). Guías para el Diagnóstico y Tratamiento de la Enfermedad Pulmonar Obstructiva CrónicaDerivadas del Cuarto Consenso Mexicano para el Diagnóstico y Tratamiento de la EPOC. Neumología y Cirugía de Tórax, 71(S1), 8-89.

UNNE. (28 de Abril de 2019). SISTEMA RESPIRATORIO. Capítulo X. Obtenido de Carrera de Licenciatura en

Enfermería: https://med.unne.edu.ar/sitio/multimedia/imagenes/ckfinder/files/files/Fisio/sistema\%20r espiratorio.p

Vargas-Domínguez, C., G.-R. L., Velázquez-Uncal, M., Mejía-Alfaro, R., Vázquez-García, J. C., Pérez-Padilla, R., \& Torre-Bouscoulet, L. (2011). Pruebas de función respiratoria,¿cuál ya quién? Neumología y Cirugía de Tórax, 70(2), 101-117.

Vinaccia, S., Quiceno, J. M., Zapata, C., Obesso, S., \& Quintero, D. C. (2011). Calidad de vida relacionada con la salud y emociones negativas en pacientes con diagnóstico de enfermedad pulmonar obstructiva crónica (EPOC). Psicología desde el Caribe, 89-108.

Walther, J. Á., Hernando, E. C., París, J. M., Toña, K. N., Aineto, P. S., Jiménez, J. M., \& ... Bañuelos, J. V. (2001). Recomendaciones para la atención al paciente con enfermedad pulmonar obstructiva crónica. Atención Primaria, 28(7), 491-500.

Zenteno, D., Puppo Gallardo, H., Vera Uribe, R., Torres, R., Kuo, C. Y., Salinas, P., \& Prado, F. (2007). Guías de rehabilitación para niños con enfermedades respiratorias crónicas. . NEUMOLOGIA PEDIATRICA, 28-33. 